\title{
Relationship between background cancer pain, breakthrough pain, and analgesic treatment: a preliminary study for a better interpretation of epidemiological and clinical studies
}

Sebastiano Mercadante, Alessandro Valle, Giampiero Porzio, Federica Aielli, Claudio Adile, Corrado Ficorella, Maurizio Raineri, Antonino Giarratano \& Alessandra Casuccio

To cite this article: Sebastiano Mercadante, Alessandro Valle, Giampiero Porzio, Federica Aielli, Claudio Adile, Corrado Ficorella, Maurizio Raineri, Antonino Giarratano \& Alessandra Casuccio (2013) Relationship between background cancer pain, breakthrough pain, and analgesic treatment: a preliminary study for a better interpretation of epidemiological and clinical studies, Current Medical Research and Opinion, 29:6, 667-671, DOI:

10.1185/03007995.2013.792247

To link to this article: http://dx.doi.org/10.1185/03007995.2013.792247

View supplementary material $\asymp$

Submit your article to this journal $₫$
Accepted author version posted online: 03 Apr 2013.

Published online: 24 Apr 2013.

III Article views: 180 


\section{Original article \\ Relationship between background cancer pain, breakthrough pain, and analgesic treatment: a preliminary study for a better interpretation of epidemiological and clinical studies}

Sebastiano Mercadante

Pain Relief and Palliative Care Unit, La Maddalena Cancer Center, Palermo, Italy

Department of Anesthesia, \& Intensive Care, University of Palermo, Palermo, Italy

Alessandro Valle

Home care program, Fondazione FARO, Torino, Turin, Italy

Giampiero Porzio

\section{Federica Aielli}

Department of Oncology, University of L'Aquila and Home care program, L'Aquila per la vita, L'Aquila, Italy

Claudio Adile

Pain Relief and Palliative Care Unit, La Maddalena Cancer Center, Palermo, Italy

\section{Corrado Ficorella}

Department of Oncology, University of L'Aquila and Home care program, L'Aquila per la vita, L'Aquila, Italy

Maurizio Raineri

Antonino Giarratano

Department of Anesthesia, \& Intensive Care,

University of Palermo, Palermo, Italy

\section{Alessandra Casuccio}

Department of Experimental Biomedicine and Clinical Neuroscience, University of Palermo, Palermo, Italy

Address for correspondence:

Dr Sebastiano Mercadante MD, Pain Relief and Palliative Care Unit, La Maddalena Cancer Center, Via San Lorenzo 312, 90146 Palermo, Italy. Tel.: +39 0916806521; Fax: +39 0916806110; terapiadeldolore@lamaddalenanet.it; 03sebelle@gmail.com

\section{Key words:}

Breakthrough pain - Cancer pain - Epidemiology Opioids

Accepted: 1 April 2013; published online: 23 April 2013 Citation: Curr Med Res Opin 2013; 29:667-71

\section{Abstract}

Background:

The different operational definitions of breakthrough cancer pain (BTcP) has generated unclear epidemiological data.

\section{Methods:}

A consecutive sample of patients was categorized on the basis of their background pain intensity, background analgesic treatment, and the presence of BTCP.

\section{Results:}

A total of 265 patients were surveyed; 117 patients had background pain and 91 patients presented peaks of pain intensity distinguishable from background pain. Of 117 patients with background pain, 49 patients were re-assessed after optimization of background analgesia ( $(1)$ within a mean of 8.2 days. Pain intensity significantly decreased in comparison with values recorded at admission $(p<0.0005) ; 75.5 \%$ of these patients had BTcP episodes, with a significant decrease in the number BTcP episodes in comparison with TO $(p<0.0005)$. The mean BTcP intensity was significantly lower in comparison with T0 $(p<0.0005)$. Finally, the mean duration of untreated BTCP episodes decreased significantly in comparison with T0 $(p=0.016)$. After optimization of analgesic therapy, most patients with moderate or severe background pain receiving opioids for moderate pain, patients with moderate or severe pain receiving strong opioids, and patients with moderate or severe pain receiving no opioids moved to the group of patients with mild pain receiving strong opioids. The difference was significant $(p=0.022)$

\section{Conclusion:}

Patients having good pain control after optimization of the analgesic regimen may have a decrease in number, intensity, and duration of BTCP, although the general prevalence of BTCP remains unchanged.

\section{Introduction}

Breakthrough cancer pain $(\mathrm{BTCP})$ has been variably reported in literature, ranging in $40-80 \%$ of cancer patients with pain, depending on the setting and the definition used to identify it ${ }^{1-4}$. The pioneer definition suggests that "BTcP is a transitory increase in pain to greater than moderate intensity which occurs on a baseline pain of moderate intensity or less" scoring, this sentence may sound ambiguous, as pain intensity should be severe (on a numerical scale 7/10), but the baseline pain could be moderate (on a numerical scale $4-6 / 10)^{6}$. Thus, the differences between the intensity of BTcP could be minimal (1-2 points on a numerical scale).

This definition was subsequently improved: "BTcP is a transitory exacerbation of pain that occurs on a background of otherwise stable pain in a patient 
receiving chronic opioid therapy" ${ }^{\prime 5}$. This sentence introduces a second variable, which is the use of stable doses of opioids able to maintain baseline pain control, although this does not mean that baseline pain is effectively controlled, allowing a clear distinction with the peak intensity of $\mathrm{BT} c \mathrm{P}$. This definition also formed the basis for a new, but similar definition: "... despite relatively stable and adequately controlled baseline pain" ${ }^{\prime, 7,8}$. Other authors have proposed that $\mathrm{BTcP}$ is a transient exacerbation of pain that occurs irrespective of basal analgesia ${ }^{9}$, episodes of pain occurring on an unrealistic pain-free background ${ }^{10}$, or "any transient flare of pain subjectively distinguishable from an otherwise more or less stable background pain"11.

In some cases there was no operational definition a priori, with incident pain due to movement given as an example, even though patients could have their pain uncontrolled or were not receiving opioids ${ }^{12}$. Baseline pain intensity was severe-maximal in more than half of patients and, differently from what has been observed by others ${ }^{12,13}$, intensity of baseline pain was higher in patients without BTcP. In other studies most patients had their pain uncontrolled, were receiving nonopioid analgesics or weak opioids, or were satisfied with their pain control ${ }^{13,14,15}$.

It is difficult to have a clear idea on a complex phenomenon without a prospective evaluation and an optimized analgesic approach. For these reasons it is likely that BTcP should be more correctly defined as an episode of severe intensity in patients receiving an adequate treatment with opioids able to provide at least mild analgesia ${ }^{4,6}$.

The aim of this study was to characterize BTcP in advanced cancer patients according to three variables, including background pain intensity, BTcP intensity, and analgesic treatment, addressing the hypothesis that improved background analgesia can modify the prevalence, frequency and intensity of BTcP.

\section{Patients and methods}

A cohort of cancer patients consecutively admitted to palliative care was surveyed in two home care palliative care units, FARO foundation (Turin) and L'Aquila per la vita (L'Aquila), respectively, for a period of 4 months. These units belong to HOCAI (Home Care Italy) group and have a similar profile in terms of assessment and treatment, providing daily visits or contacts with a complete staff of physicians, nurses, psychologists, and social workers with consolidated experience in palliative care and in collecting data for research.

Demographic characteristics were recorded. Patients were assessed for the presence of background pain and its intensity in the last 24 hours, as well as the intensity of BTcP, intended as an episode of severe pain intensity $(\geq 7 / 10)$ well distinguished from the background pain intensity. This definition was shared by researchers in a preliminary investigator meeting. The subgroup of patients with $\mathrm{BTcP}$ due to movement in patients with bone metastases was also identified. Analgesic treatment was recorded.

At admission, patients were divided into different categories, according to the level of background pain intensity and analgesic drugs used at admission (TO):

(a) Patients with no pain-mild pain without opioids

(b) Patients with mild pain receiving weak opioids

(c) Patients with moderate pain receiving weak opioids

(d) Patients with severe pain receiving weak opioids

(e) Patients with mild pain receiving strong opioids

(f) Patients with moderate pain receiving strong opioids

(g) Patients with severe pain receiving strong opioids

(h) Patients with severe pain, receiving no opioids

(i) Patients with moderate pain, receiving no opioids.

In patients with background analgesia which was considered inadequate (mainly with a background pain $>4 / 10$ on a numerical scale $0-10$ ), the same data were collected after changing the analgesic treatment until a satisfactory analgesia was achieved $(\leq 4 / 10)$.

Treatment was decided by the responsible physician from the home care team. The analgesic treatment consisted of the optimization of opioid doses, eventually changing opioids, while limiting opioid-related symptoms with adequate treatment.

Pain and symptom intensity were continuously monitored day-by-day, and treatment changed until the best balance between analgesia and adverse effects was achieved. Patients were divided into the same categories at this time (T1). Other data regarding the characteristics of $\mathrm{BT} c \mathrm{P}$ were collected (see appendix for details). Patients were divided into two groups: advanced cancer patients receiving chemotherapy, and very advanced cancer patients no longer receiving chemotherapy (with a survival of less than 2 months).

\section{Statistics}

All continuous data are expressed as mean \pm standard deviation of the mean. Statistical analysis of quantitative data, included descriptive statistics, was performed for all the items. Frequency analysis was performed using Pearson's chi-square test and Fisher's exact test, as needed. The paired samples Student's t-test and the paired Wilcoxon signed-rank test were used to compare parametric and nonparametric variables, respectively, at the different intervals. Data were analyzed using Epi Info software, version 3.2.2 (Centers for Disease Control and Prevention) and SPSS Software version 14.0 (SPSS Inc., Chicago, IL, USA). All p-values were two sided, and $p$-values less than 0.05 were considered statistically significant. 


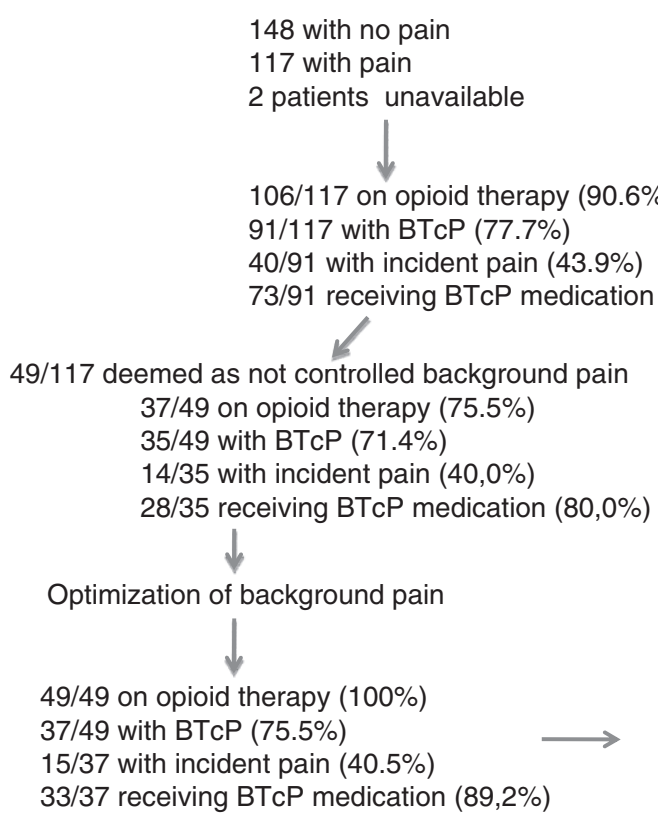

148 with no pain

117 with pain

2 patients unavailable

$106 / 117$ on opioid therapy (90.6\%)

$91 / 117$ with BTcP $(77.7 \%)$

40/91 with incident pain (43.9\%)

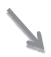

$68 / 117$ with controlled pain

$57 / 68$ on opioid therapy (83.8\%)

$54 / 68$ with BTcP $(79.4 \%)$

$25 / 54$ with incident pain (46.3\%)

40/54 receiving BTcP medication (74,1\%)

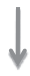

117 with controlled pain

106 on opioid therapy $(90.5 \%)$

91 with BTcP $(77.7 \%)$

40 with incident pain (43.9\%)

77 receiving $\mathrm{BTcP}$ medication (84.6\%)

Figure 1. Study flow chart.

\section{Results}

A total of 267 patients entered the study and 265 patients were surveyed (two patients were too ill and unable to participate). The mean age was 67.7 years $(S D \pm 14.2)$, and $107(40.4 \%)$ were males. Primary tumors were in a rank order: gastrointestinal $(n=64)$, urogenital $(n=48)$, lung $(n=47)$, breast $(n=45)$, head and neck $(n=17)$, liver $(n=11)$, pancreas $(n=11)$, and others $(n=22)$. The mean Karnofsky performance status was 69.8 $(\mathrm{SD} \pm 17.4)$. The flow chart of patients who were surveyed is presented in Figure 1.

A total of 148 patients had no lasting pain for most hours of day; 117 patients (44.1\%) had background pain with a mean intensity at admission of $3.6(\mathrm{SD} \pm 2.4) ; 106$ of them (90.6\%) were receiving opioids; 91 patients $(77.7 \%)$ presented peaks of pain intensity distinguishable from background pain, and 40 of them $(43.9 \%)$ had pain on movement due to bone metastases, interfering with daily life. The mean pain intensity of these episodes was $8.5(\mathrm{SD} \pm 2.6)$, the duration of untreated episodes was 28.8 minutes $(\mathrm{SD} \pm 25.7)$, and the mean number of episodes was $2.7 /$ day $(\mathrm{SD} \pm 1.4)$. Seventy-three patients $(80.2 \%)$ were receiving opioids for BTcP and efficacy was high, moderate, and poor in 32,33 , and 8 patients, respectively.

Out of 117 patients $49(53.8 \%)$ were considered to have uncontrolled background pain (mean pain intensity 6.1, $\mathrm{SD} \pm 1.6$ ); 37 patients were receiving opioids, and 35 had episodes distinguished from background analgesia. Of these, 14 patients had bone pain due to movement. Twenty-eight patients were receiving BTcP medication.
Twenty-eight patients with $\mathrm{BTcP}(80 \%)$ were prescribed opioids for BTcP, which were considered to be highly, moderately, and poorly effective in 7,17 , and 4 patients, respectively.

In these 49 patients the analgesic treatment or doses of opioids were changed and a re-assessment was performed after obtaining adequate background analgesia (T1) within a mean of 8.2 days $(\mathrm{SD} \pm 3.7)$. At $\mathrm{T} 1$ the mean background pain intensity of these 49 patients was 1.9 $(\mathrm{SD} \pm 1.2)$, with a significant decrease in pain intensity in comparison with values recorded at TO $(p<0.0005)$. Thirty-seven of these patients $(75.5 \%)$ had BTcP episodes, with a significant decrease in the number of BTcP episodes in comparison with $\mathrm{TO}(3.2, \mathrm{SD} \pm 1.7$, at $\mathrm{T} 0 \mathrm{vs}$ $2.0 \pm 0.8$ at $\mathrm{T} 1, p<0.0005)$. Fifteen of them $(15 / 37$, $40.5 \%$ ) presented bone pain due to movement, interfering with daily life in 13 patients. The mean BTcP intensity was $8.0(\mathrm{SD} \pm 1.15)$, which decreased significantly in comparison with T0 $(9.0, \mathrm{SD} \pm 1.1 ; p<0.0005)$. Finally, the mean duration of untreated BTCP episodes was 29 minutes $(\mathrm{SD} \pm 23.7)$, which decreased significantly in comparison with TO (39.2, SD $\pm 32.2 ; p=0.016)$. Thirty-three patients with BTcP $(89.2 \%)$ were prescribed opioids for $\mathrm{BTcP}$, which were considered to be highly, moderately, and poorly effective in 10,22 , and 1 patients, respectively. The difference was not significant $(p=0.275)$.

The number of patients belonging to categories A-I (see methods), and the prevalence of BTcP in these categories at admission (TO), are presented in Table 1. Data regarding the 49 patients with uncontrolled pain at admission (TO) and after optimization of analgesic treatment 
Table 1. Classification of patients according to their pain intensity and analgesic treatment (see text), and prevalence of BTcP.

\begin{tabular}{lcc}
\hline & $N=265$ & $\operatorname{BTcP}(n=91)$ \\
\hline A & $148(55.8 \%)$ & 0 \\
B & $36(13.6 \%)$ & 27 \\
C & $8(3 \%)$ & 8 \\
D & $7(2.6)$ & 7 \\
E & $35(13.2 \%)$ & 3 \\
F & $12(4.5 \%)$ & 10 \\
G & $8(3 \%)$ & 8 \\
H & $5(1.9 \%)$ & 0 \\
I & $6(2.3 \%)$ & 0 \\
\hline
\end{tabular}

Ninety-one of 117 of patients with some pain had BTcP $(78 \%)$.

Table 2. Data regarding patients with uncontrolled pain at admission (T0) and after optimization of analgesic treatment, and changes of BTCP prevalence $(p=0.022)$.

\begin{tabular}{lrrrrr}
\hline & \multicolumn{2}{c}{$N=49$} & & \multicolumn{2}{c}{ BTP $(n=37,75.5 \%)$} \\
\cline { 2 - 3 } \cline { 5 - 6 } & T0 & T1 & & T0 & T1 \\
\hline A & 1 & 1 & & 0 & 0 \\
B & 1 & 17 & & 1 & 12 \\
C & 8 & 0 & & 8 & 0 \\
D & 7 & 1 & & 7 & 1 \\
E & 1 & 30 & & 1 & 24 \\
F & 12 & 0 & & 9 & 0 \\
G & 8 & 0 & & 5 & 0 \\
H & 5 & 0 & & 3 & 0 \\
I & 6 & 0 & & 3 & 0 \\
\hline
\end{tabular}

First columns: Categorization A-I (see text) of the 49 patients with uncontrolled pain at admission (T0) and after optimization of analgesic treatment (T1). Second columns: data regarding the changes in BTcP prevalence between T0 and T1 among the different categories $(p=0.022)$.

(T1) in the different categories A-I, and data regarding the changes of BTcP prevalence between $\mathrm{TO}$ and $\mathrm{T} 1$ among the different categories A-I, are presented in Table 2 . Most patients with BTcP included in categories $\mathrm{C}$ and $\mathrm{D}$ (patients with moderate or severe pain receiving opioids for moderate pain), $F$ and $G$ (patients with moderate or severe pain receiving strong opioids), $\mathrm{H}$ and I (patients with moderate or severe pain receiving no opioids) moved to category E (patients with mild pain receiving strong opioids), after optimizing analgesic treatment. The difference was significant $(p=0.022)$. No differences between patients receiving chemotherapy and patients no longer receiving treatment was observed at either $\mathrm{T} 0$ or $\mathrm{T} 1, p=0.506$ and 0.181 , respectively.

\section{Discussion}

Background pain intensity, background analgesic treatment, and intensity of $\mathrm{BTCP}$ have never been concomitantly assessed in epidemiological and clinical studies of $\mathrm{BT}$ cP. In some studies, peaks of pain intensity have been reported independently on the analgesic treatment, and in others no clear distinction between background and BTcP pain intensity has been reported ${ }^{10-14,16,17}$. We have previously shown that many studies of BTcP have been performed in patients with uncontrolled background pain. The gray range of moderate pain (5-6/10) has been indifferently included in the literature as belonging to both baseline pain or $\mathrm{BTcP}$, and in some cases patients with severe background pain were included ${ }^{18,19}$, rendering the interpretation of data problematic. In most epidemiological surveys, patients not stabilized on adequate opioid therapy have been considered as patients with BTcP. Patients with uncontrolled background pain not clearly distinguished from peaks of pain intensity should be not considered as ideal for either epidemiological studies or assessing medication for BTcP. These patients should also have their pain controlled, which is conventionally considered to be $\leq 4 / 10$ on a numerical scale $0-10^{6,20}$.

In this study, BTcP prevalence was examined in a global number of patients who would have been included for the determination of $\mathrm{BTcP}$ and in a subgroup of patients who, in our opinion, had insufficient background analgesia and required an optimization of the analgesic treatment. This approach produced not only an improvement in background pain intensity, but also a decrease in the number of episodes/day of BTcP, a decreased intensity and duration. Patients with moderate (category F) and severe background pain (category $G$ ) receiving opioids moved to other categories (substantially category E) after optimization of therapy, explaining the improvement in $\mathrm{BTCP}$ characteristics and confirming previous observations which reported that BTcP may change after 1 week of opioid titration $^{21,22}$.

The global prevalence of $\mathrm{BTcP}$, however, did not change despite optimization of therapy as more than $75 \%$ with adequate background pain treated with opioids may still develop BTcP despite a more moderate presentation, in terms of number, intensity, and duration.

In a more recent study, the definition included patients with background pain adequately controlled presenting episodes of $\mathrm{BTCP}_{\mathrm{C}} \mathrm{2}^{23}$. Despite a more strict selection to characterize patients with $\mathrm{BTcP}$, information regarding the ratio between the presence of pain, the intensity of background pain and $\mathrm{BTcP}$, as well as pain treatment, was not shown. Forty-four percent of patients reported BTcP related to identified factors, $39 \%$ had idiopathic BTcP, and $17 \%$ a combination of these. Almost all patients had mild background pain and severe BTcP pain was reported in $60 \%$ of patients. However, $40 \%$ of patients had mildmoderate $\mathrm{BTcP}$ pain, which is not clearly distinguished from background pain. Moreover, prevalence in the general population was not reported 4 .

The findings of the present study may be limited in terms of number of patients, especially in the subgroup of patients requiring optimization of therapy, but has the 
advantage of homogeneous and shared protocols of assessment and therapeutic interventions at home, which may often be lacking in larger multicenter studies.

In conclusion, for a clear interpretation of $\mathrm{BTcP}$, it is necessary to have well controlled background pain, with adequate opioid analgesia, and $\mathrm{BTcP}$ episodes of a certain intensity which should be clearly distinguished from background pain intensity. It has recently been found that meaningful pain intensity for asking for a BTcP medication was 7.1 , with $77 \%$ of patients having a pain intensity of 7-8 on a numerical scale $0-10^{24}$. This aspect has obvious implications from both the epidemiological and therapeutic point of view. An improvement in background analgesia, however, is unable to limit the global prevalence of $\mathrm{BTcP}$ in advanced cancer patients, but may help the treatment in terms of number of episodes to treat and efficacy of BTcP medications. Multicenter studies with a larger number of patients are necessary to confirm this preliminary observation.

\section{Transparency}

\section{Declaration of funding}

No funding sources supported this work.

\section{Declaration of financial/other relationships}

S.M., A.V., G.P., F.A., C.A., C.F., M.R., A.G., and A.C. have disclosed that they have no significant relationships with or financial interests in any commercial companies related to this study or article.

CMRO peer reviewers may have received honoraria for their review work. The peer reviewers on this manuscript have disclosed that they have no relevant financial relationships.

\section{Acknowledgements}

The authors thank and are indebted to all the staff of home care programs in Turin and L'Aquila, Italy.

\section{References}

1. Haugen D, Hjermstad M, Hagen N, et al. Assessment and classification of cancer breakthrough pain: a systematic literature review. Pain 2010; 149:476-82

2. Mercadante S, Costanzo BV, Fusco F, et al. Breakthrough pain advanced cancer patients: a longitudinal study. Support Care Cancer 2009;38:554-60

3. Mercadante S, Zagonel V, Breda E, et al. Breakthrough pain in oncology: a longitudinal study. J Pain Symptom Manage 2010;40:183-90

4. Davies A, Zeppetella G, Andersen S, et al. Multi-centre European study of breakthrough cancer pain: pain characteristics and patient perceptions of current and potential management strategies. Eur J Pain 2011;15:756-63
5. Portenoy RK, Hagen NA. Breakthrough pain: definition, prevalence and characteristics. Pain 1990:41:273-81

6. Serlin RC, Mendoza TR, Nakamura Y, et al. When is cancer pain mild, moderate or severe? Grading pain severity by its interference with function. Pain 1995;61:277-84

7. Bennett D, Burton AW, Fishman S, et al. Consensus panel recommendations for the assessment and management of breakthrough pain. Part 2 management. Pharm Ther 2005;30:354-61

8. Hagen NA, Stiles C, Nekolaichuk C, et al. The Alberta breakthrough pain assessment tool for cancer patients. A validation study using a Delphi process and patient think-aloud interview. J Pain Symptom Manage 2008;35:136-52

9. Svendsen KB, Andersen S, Arnason S, et al. Breakthrough pain in malignant and non-malignant diseases: a review of prevalence, characteristics and mechanisms. Eur J Pain 2005;9:195-206

10. Swanwick M, Haworth M, Lennard RF. The prevalence of episodic pain in cancer: a survey of hospice patients on admission. Palliat Med 2001;15:9-18

11. Petzke F, Radbruch $L$, Zech D, et al. Temporal presentation of chronic cancer pain: transitory pains on admission to a multidisciplinary pain clinic. J Pain Symptom Manage 1999;17:391-401

12. Caraceni $A$, Martini $C$, Zecca $E$, et al. Working group of an IASP task force on cancer pain. Breakthrough pain characteristics and syndromes in patients with cancer pain. An international survey. Palliat Med 2004;18:177-83

13. Gómez-Batiste $X$, Madrid $F$, Moreno $F$, et al. Breakthrough cancer pain: prevalence and characteristics in patients in Catalonia, Spain. J Pain Symptom Manage 2002; 24:45-52

14. Zeppetella G, O'Doherty CA, Collins S. Prevalence and characteristics of breakthrough pain in cancer patients admitted to a hospice. J Pain Symptom Manage 2000;20:87-92

15. Mercadante S. Pharmacotherapy for breakthrough cancer pain. Drugs 2012;72:181-90

16. Greco MT, Corli 0, Montanari M, et al. Epidemiology and pattern of care of breakthrough cancer pain in a longitudinal sample of cancer patients. Results from the Cancer Pain Outcome Research Study Group. Clin J Pain $2011 ; 27: 9-18$

17. Caraceni A, Bertetto O, Labianca R, et al. Episodic (breakthrough) pain prevalence in a population of cancer pain patients. Comparison of clinical diagnoses with the QUDEI - Italian questionnaire for intense episodic pain. J Pain Symptom Manage 2012;43:833-41

18. Mercadante S. Breakthrough pain: on the road again. Eur J Pain 2009;13: 329-30

19. Mercadante $\mathrm{S}$. The use of rapid onset opioids for breakthrough cancer pain: the challenge of its dosing. Crit Rev Oncol Hematol 2011;80:460-5

20. Corli O, Montanari M, Greco MT, et al. How to evaluate the effect of pain treatments in cancer patients: results from a longitudinal outcomes and endpoint Italian cohort study. Eur J Pain 2012: published online 5 December 2012, doi: 10.1002/j.1532-2149.2012.00257.x

21. Hwang SS, Chang VT, Kasimis B. Cancer breakthrough pain characteristics and responses to treatment at a VA medical center. Pain 2003;101: 55-64

22. Mercadante S, Villari P, Ferrera P, Casuccio A. Optimization of opioid therapy for preventing incident pain associated with bone metastases. J Pain Symptom Manage 2004;28:505-10

23. Davies A, Dickman A, Reid C, et al. The management of cancer-related breakthrough pain: recommendations of a task group of the Association of Palliative Medicine of Great Britain and Ireland. Eur J Pain 2009:13:331-8

24. Mercadante S, Adile C, Torta R, et al. Meaningful cut-off pain intensity for breakthrough pain changes in advanced cancer patients. Curr Med Res Opin 2013;29:93-7 